Part of Journal of Research of the National Bureau of Standards, Volume 31,

\title{
AXIAL RIGIDITY OF PERFORATED STRUCTURAL MEMBERS
}

\section{By Martin Greenspan}

\begin{abstract}
Formulas are derived for computing the over-all lengthening (or shortening) of a tension (or compression) member having a uniform gross cross section and a series of similar perforations of circular, elliptical, or "ovaloid" shape uniformly distributed along the length.

Tests made on strips having circular perforations show that the applicable formula gives good results over the practical range of the variables.
\end{abstract}

\section{CONTENTS}

Page

I. Introduction

II. A reduction of the problem

III. Circular and ovaloid perforations

1. Kirsch's problem

(a) The displacements _... _.

(b) The load _... 309

2. Axial rigidity for circular perforations

3. Axial rigidity for ovaloid perforations.

IV. Elliptical perforations_____

1. General _._. 312

2. Curvilinear coordinates_..__._.

3. Elliptical coordinates

4. Inglis' problem for plane stress

5. Axial rigidity for load parallel to major axis of ellipse _._. _ _ _ _ 318

6. Axial rigidity for load parallel to minor axis of ellipse $\ldots \ldots \ldots$

V. Tests on tension members having circular perforations_____

1. General

2. Description _.

3. Results

VI. Summary

VII. References

\section{INTRODUCTION}

The work reported in this paper is part of an investigation at the National Bureau of Standards on perforated cover plates. The results of tests on a large number of columns having perforated cover plates have been reported [1]. ${ }^{1} \quad$ From these results may be computed a value for each perforated column of an "axial rigidity factor", $K$, which is the ratio of the axial rigidity of the column to the axial rigidity of an

1 Figures in brackets indicate the literature references at the end of this paper. 
unperforated, but otherwise similar, column. The axial rigidity, $R$, of a tension or compression member of length $L$ is defined from

$$
e=\frac{P L}{R},
$$

where $P$ is the axial load and $e$ the lengthening or shortening of the member, and $P / e$ is constant. For a member of uniform cross-sectional area $A_{\imath}, R=E A_{\imath}$, where $E$ is Young's modulus of elasticity.

In this paper are derived formulas for computing the axial-rigidity factor of a member having a uniform gross cross section and a series of similar perforations of circular, elliptical, or "ovaloid" shape uniformly distributed along the length. Such formulas may find application whenever information concerning displacements in a structure having perforated members is required. Examples are the analysis of indeterminate structures and the computation of the camber of trusses.

The results of some tests on tension members having circular perforations are reported and compared with the values computed from the applicable formula.

\section{A REDUCTION OF THE PROBLEM}

Consider a long tension (or compression) member of uniform gross cross section, containing a series of similar perforations spaced uniformly along the length. The member is supposed subjected to an axial load, $P$, and it is desired to study the over-all deformation in the axial direction produced by the load.

For this purpose the member is considered divided into similar bays of length $2 l$, each bay containing one perforation. If the perforation has symmetry about an axis perpendicular to that of the tension member, the analysis may be confined to a half-bay of length $l$. The problem is now to determine the over-all extension of the half-bay when subjected to a load, $P$, distributed over the net area, $A_{n}$, on one end, and over the gross area, $A_{g}$, on the other end, in such a manner that these ends remain plane (fig. 1 , a).

With the $x$-axis taken parallel to the outer elements of the member, and the origin of coordinates on the transverse axis of the perforation, the problem is to find the displacement, $u_{l}$, of the end $x=l$, that of the end $x=0$ being taken as zero. The $x$-components of the displacements of points on the bounding surface of the perforation will be denoted by $u_{p}$.

Consider, in addition, a state of stress in the member consisting of a uniform normal stress $\sigma_{x}=P / A_{g}$ throughout, all other stresses being zero (fig. 1, b). This stress could be maintained by surface loads $\pm P / A_{g}$ per unit of projected (on the $y z$-plane) area, positive on the right end and negative on the left end, of the member. The displacement of the end $x=l$ is $P l / A_{g} E$.

To these two states of stress, namely (a) that prevailing in the original problem, and (b) that characterized by a uniform stress, the reciprocal theorem ${ }^{2}$ may be applied. This theorem states that the work done by the external forces which correspond to the first state acting through the displacements which correspond to the second state is equal to the work done by the external forces which corre-

\footnotetext{
2 See p. 173 of reference [2].
} 
spond to the second state acting through the displacements which correspond to the first state. Hence

$$
\frac{1}{2} P u_{l}-\frac{1}{2} \int \frac{P}{A_{g}} u_{p} d y d z=\frac{1}{2} \frac{P^{2} l}{A_{g} E}
$$

or

$$
u_{l}=\frac{P l}{A_{g} E}+\frac{1}{A_{z}} \int u_{p} d y d z
$$

where the integration is to be taken over the projection on the $y z$ plane of the bounding surface of the perforation.

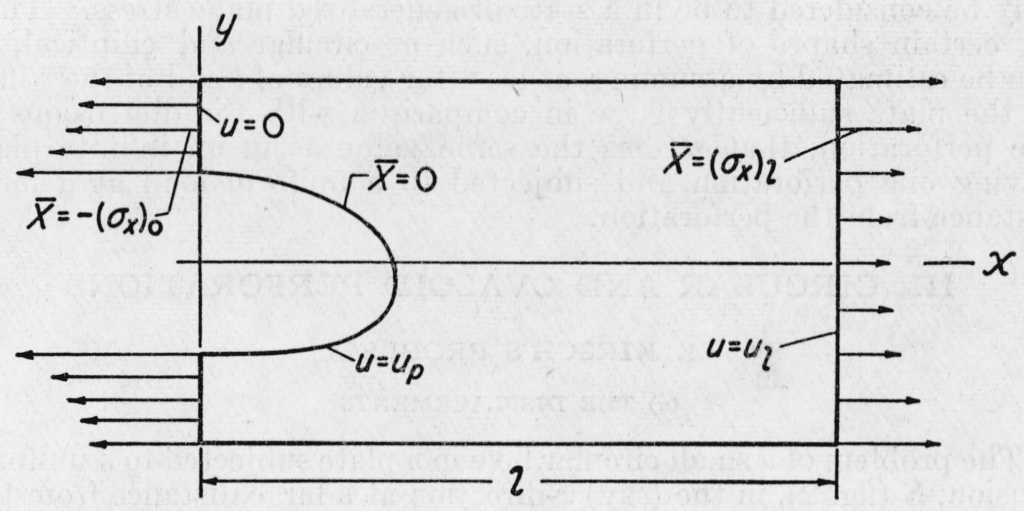

(a)

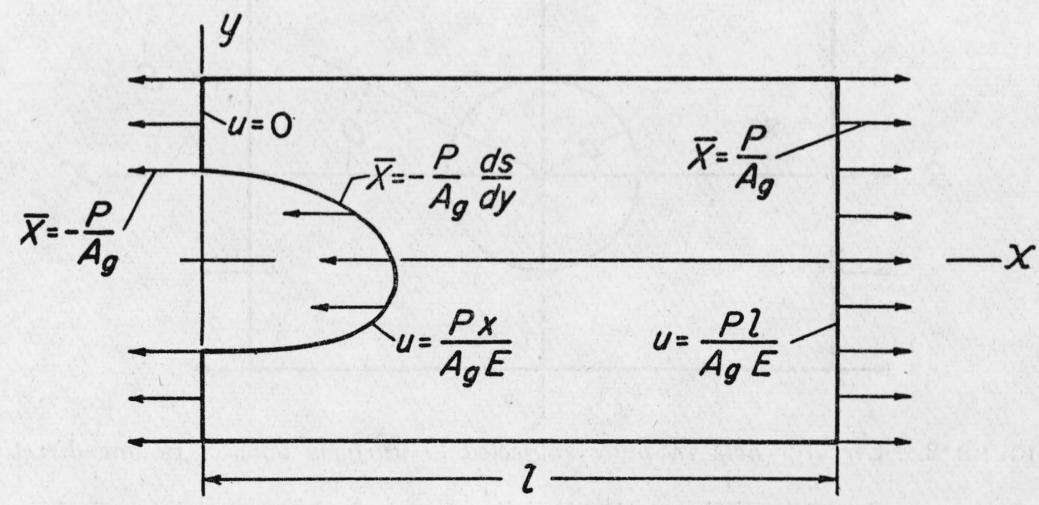

(b)

Figure 1.-Two states of stress in a half-bay of the member.

(a) The cross sections $x=0$ and $x=l$ remain plane; (b) uniform stress, in the axial direction, throughout.

In an unperforated member of the same gross dimensions and subjected to the same load as the perforated half-bay, the displacement, $u^{\prime}{ }_{l}$, of the end $x=l$ is

$$
u^{\prime}=\frac{P l}{A_{8} E}
$$


The ratio of the axial rigidity of the perforated member to that of the unperforated member is the same as the ratio of these displacements. Hence

$$
K=\frac{u_{l}^{\prime}}{u_{l}}=\frac{1}{1+\frac{E}{P l} \int u_{p} d y d z}
$$

Equation 2 enables the computation of $K$, provided the values of $u_{p}$ can be determined. If the problem be restricted to the case where the perforation occurs in a relatively thin plate of constant thickness, $t$, then the material in the neighborhood of the perforation may be considered to be in a state of generalized plane stress. Then for certain shapes of perforation, such as circular and elliptical, $u_{p}$ can be estimated by assuming, at least for values of $l$ and of the width of the plate sufficiently large in comparison with the dimensions of the perforation, that $u_{p}$ has the same value as in an infinite plate having one perforation and subjected to a uniform load at a large distance from the perforation.

\section{CIRCULAR AND OVALOID PERFORATIONS}

\section{KIRSCH'S PROBLEM}

(a) THE DISPLACEMENTS

The problem of a small circular hole in a plate subjected to a uniform tension, $S$ (fig. 2), in the (say) $x$-direction at a large distance from the

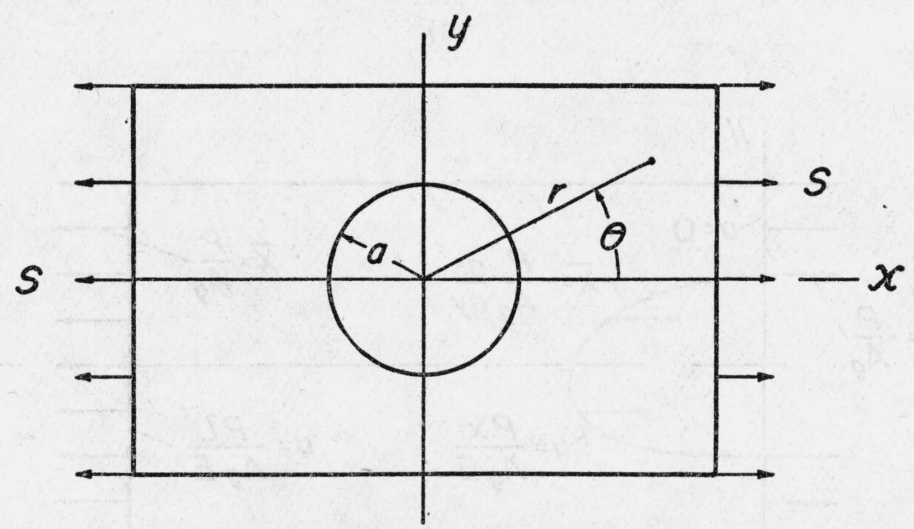

Figure 2.-Circular hole in plate subjected to uniform tension in one direction.

hole was solved by Kirsch [3] for the case of plane strain. It is convenient to solve for the displacements in the case of plane stress by a method $^{3}$ depending on a knowledge of the Airy stress function, $\phi$. The function, $\psi$, defined, in the absence of body forces, by

$$
\frac{\partial^{2} \psi}{\partial x \delta y}=\frac{\partial^{2} \phi}{\partial x^{2}}+\frac{\partial^{2} \phi}{\partial y^{2}}=\nabla^{2} \phi
$$

with $\psi$ adjusted by means of the arbitrary functions of integration (of

${ }^{3}$ See p. 130 of reference [4]. 
the form $\left.f_{1}(x)+f_{2}(y)\right)$, so that $\nabla^{2} \psi=0$, is called the displacement function. The $x$-component of the displacement is given by

$$
u=\frac{1}{E}\left[\frac{\partial \psi}{\partial y}-(1+\nu) \frac{\partial \phi}{\partial x}\right]
$$

where $\nu$ is Poisson's ratio.

The solution to Kirsch's problem is given by the stress function ${ }^{4}$

$$
\phi=\frac{S}{4}\left[r^{2}-2 a^{2} \log r-\frac{\left(r^{2}-a^{2}\right)^{2}}{r^{2}} \cos 2 \theta\right],
$$

where $a$ is the radius of the circle (fig. 2), so that

$$
\nabla^{2} \phi=\frac{\partial^{2} \psi}{\partial x \partial y}=S\left(1-\frac{2 a^{2}}{r^{2}} \cos 2 \theta\right)=S\left[1-2 a^{2} \frac{x^{2}-y^{2}}{\left(x^{2}+y^{2}\right)^{2}}\right]
$$

From which

and

$$
\frac{\psi}{S}=x y-2 a^{2} \tan ^{-1} \frac{x}{y}+c\left(x^{2}-y^{2}\right)+d x+e y+f,
$$

$$
\frac{1}{S} \frac{\partial \psi}{\partial y}=x+\frac{2 a^{2} x}{x^{2}+y^{2}}-2 c y+e .
$$

The primitive of $\psi$ is harmonic, so that the arbitrary functions of integration represented by the last four terms in eq 5 are merely the most general harmonic function of the form $f_{1}(x)+f_{2}(y)$.

From eq 4

$$
\frac{\partial \phi}{\partial x}=\frac{S}{2} a^{2} x \frac{x^{2}+y^{2}-a^{2}}{\left(x^{2}+y^{2}\right)^{3}}\left(3 y^{2}-x^{2}\right) .
$$

Substitution of $\partial \psi / \partial y$ from eq 6 and $\partial \phi / \partial x$ from eq 7 in eq 3 gives

$$
u=\frac{S}{E}\left[x+\frac{2 a^{2} x}{x^{2}+y^{2}}-2 c y+e-\frac{1+\nu}{2} a^{2} x \frac{x^{2}+y^{2}-a^{2}}{\left(x^{2}+y^{2}\right)^{3}}\left(3 y^{2}-x^{2}\right)\right] .
$$

The condition $u=0$ for $x=0$ gives $c=e=0$, so that finally,

$$
u=\frac{S}{E}\left[x+\frac{2 a^{2} x}{x^{2}+y^{2}}-\frac{1+v}{2} a^{2} x \frac{x^{2}+y^{2}-a^{2}}{\left(x^{2}+y^{2}\right)^{3}}\left(3 y^{2}-x^{2}\right)\right]
$$

For points on the circle $x^{2}+y^{2}=a^{2}$, eq 8 becomes

$$
u_{p}=\frac{3 S x}{E} \text {. }
$$

Equation 9 gives the value of $u_{p}$ that should be used in eq 2 to obtain $K$.

(b) THE LOAD

It remains to determine the value of $P$ in eq 2. Consider the strip bounded by the lines $y= \pm n a$. If $t$ is the thickness of the plate, the 
total load carried by the strip is $P^{\prime}=2 \int_{0}^{n a} \sigma_{x} t d y$. This load is a function of $x / a$, and will vary along the length of the strip from $x=a$ to $x=l$. However, this variation is small for sufficiently large $n$, as is easily shown. From eq 4

$$
\begin{aligned}
& \sigma_{x}=\frac{\partial^{2} \phi}{\partial y^{2}}=S\left[1-\frac{5 a^{2}}{2\left(x^{2}+y^{2}\right)}+\frac{a^{2}\left(2 y^{2}+3 a^{2}\right)}{2\left(x^{2}+y^{2}\right)^{2}}\right. \\
& \left.-\frac{4 a^{2} y^{2}\left(2 y^{2}+3 a^{2}\right)}{\left(x^{2}+y^{2}\right)^{3}}+\frac{12 a^{4} y^{4}}{\left(x^{2}+y^{2}\right)^{4}}\right]
\end{aligned}
$$

and with the notation, $x=m a$,

$$
P^{\prime}=2 \int_{0}^{n a} \sigma_{x} t d y=2 \text { Snat }\left[1-\frac{1}{2\left(m^{2}+n^{2}\right)}-\frac{4 m^{2}+1}{2\left(m^{2}+n^{2}\right)^{2}}+\frac{2 m^{2}}{\left(m^{2}+n^{2}\right)^{3}}\right] .
$$

From eq 10 it may be computed that for given $n, P^{\prime} / 2$ Snat will vary from its value at $m=0$ by not more than 2 percent for the following values of $n$ and $m$ :

$$
\begin{array}{ll}
n=3, & m \ngtr 6 \\
n=4, & m \ngtr 13 \\
n>5, & \text { any } m .
\end{array}
$$

Hence $P^{\prime}$ may be taken as its value at $m=0$, that is, as the load transmitted by the strip across the minimum cross section, or

$$
P^{\prime}=2 \operatorname{Snat}\left(1-\frac{1}{2 n^{2}}-\frac{1}{2 n^{4}}\right)
$$

$P^{\prime}$ may be used for $P$ in eq 2 if $n$ be interpreted as

$$
A_{g} / 2 a t=\frac{A_{g}}{A_{g}-A_{n}}
$$

hence

$$
P=S A_{g}\left(1-\frac{1}{2 n^{2}}-\frac{1}{2 n^{4}}\right)=S A_{g} C(n),
$$

say. $C(n)$ may be considered to be a correction factor depending on $n$.

2. AXIAL RIGIDITY FOR CIRCULAR PERFORATIONS

Substitution of $u_{p}$ from eq 9 and $P$ from eq 11 in eq 2 gives

$$
K=\frac{1}{1+\frac{3}{C(n)} \frac{1}{A_{g} l} \int x t d y}
$$

The integral above is one-half of $V_{0}$ the volume of the perforation, and $A_{\theta} l$ is one-half of $V_{\theta}$, the gross volume of one bay of the tension member. Hence 


$$
K=\frac{1}{1+\frac{3}{C(n)} \frac{V_{0}}{V_{g}}} .
$$

\section{AXIAL RIGIDITY FOR OVALOID PERFORATIONS}

An "ovaloid" perforation is one having the shape of a rectangle with a semicircle erected on each end. Consider a tension member having an ovaloid perforation with dimensions as shown in figure 3 and with

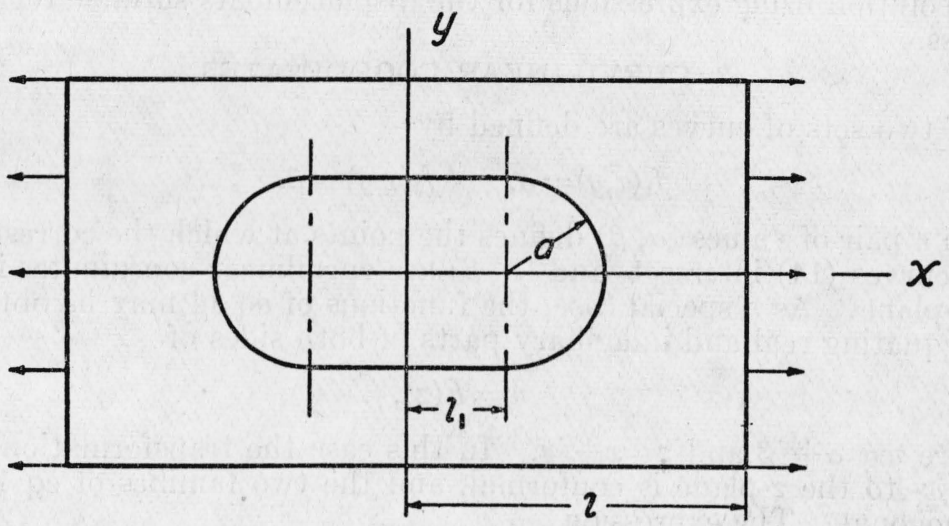

FigURE 3.-Ovaloid hole in plate subjected to uniform tension parallel to long axis of ovaloid.

the long axis of the perforation in the direction of the load. As an approximation, $u_{p}$ may be taken as its value in Kirsch's problem plus $P l_{1} / A_{n} E$, that is,

$$
u_{p}=\frac{P l_{1}}{A_{n} E}+\frac{3 P\left(x-l_{1}\right)}{C(n) A_{g} E}=\frac{P}{E A_{0}}\left[\frac{A_{g}}{A_{n}} l_{1}+\frac{3}{C(n)}\left(x-l_{1}\right)\right] .
$$

Substitution of this value of $u_{p}$ in eq 2 gives

$$
K=\frac{1}{1+\frac{1}{A_{g} l} \int\left[\frac{A_{g}}{A_{n}} l_{1}+\frac{3}{C(n)}\left(x-l_{1}\right)\right] t d y} .
$$

As before, $A_{\theta} l$ is one-half of $\mathrm{V}_{g}$, the gross volume of one bay; $\int l_{1} t d y$ is one-half of $V_{r}$, the volume of the rectangular portion of the perforation; and $S\left(x-l_{1}\right) t d y$ is one-half of $\mathrm{V}_{c}$, the volume of the circular portion of the perforation. Hence

$$
K=\frac{1}{1+\frac{A_{g}}{A_{n}} \frac{V_{r}}{V_{g}}+\frac{3}{C(n)} \frac{V_{c}}{V_{g}}} .
$$




\section{ELLIPTICAL PERFORATIONS}

\section{GENERAL}

The problem of a small elliptical hole in a plate subjected to uniform tension, $S$, in any direction at a large distance from the hole was solved by Inglis [5]. The method of Inglis amounts to assuming expressions for the displacements in the case of plane strain and determining constants to satisfy the state of stress at the boundary. The solution as given is not directly applicable to the determination of the displacements in the case of plane stress; hence it is proposed to repeat the solution using expressions for the displacements suitable for plane stress.

\section{CURVILINEAR COORDINATES}

If two sets of curves are defined by

$$
f_{1}(x, y)=\alpha, \quad f_{2}(x, y)=\beta,
$$

then a pair of values $(\alpha, \beta)$ defines the points at which the corresponding curves (14) intersect, and $(\alpha, \beta)$ are curvilinear coordinates in the $x, y$-plane. As a special case, the functions of eq 14 may be obtained by equating real and imaginary parts of both sides of

$$
w=F(z),
$$

where $w=\alpha+i \beta$ and $z=x+i y$. In this case the transformation from the $w$ - to the $z$-plane is conformal, and the two families of eq 14 are orthogonal. The expression

$$
\frac{d z}{d w}=\frac{1}{h} e^{\imath \phi},
$$

defines the "stretch ratio," $1 / h$, of the transformation, and gives $\phi$, the inclination of the curve, $\beta=$ constant, to the $x$-axis.

If $u_{\alpha}$ and $u_{\beta}$ are the components of the displacement in the directions $\alpha$-increasing, $\beta$-increasing, respectively, and

$$
u=u_{\alpha} / h, \quad v=u_{\beta} / h,
$$

the components of strain are

$$
\left.\begin{array}{c}
\epsilon_{\alpha}=h^{2} \frac{\partial u}{\partial \alpha}+\frac{u}{2} \frac{\partial h^{2}}{\partial \alpha}-\frac{v}{2} \frac{\partial h^{2}}{\partial \beta}, \\
\epsilon_{\beta}=h^{2} \frac{\partial v}{\partial \beta}+\frac{v}{2} \frac{\partial h^{2}}{\partial \beta}-\frac{u}{2} \frac{\partial h^{2}}{\partial \alpha}, \\
\gamma_{\alpha \beta}=h^{2}\left(\frac{\partial v}{\partial \alpha}+\frac{\partial u}{\partial \beta}\right)+u \frac{\partial h^{2}}{\partial \beta}+v \frac{\partial h^{2}}{\partial \alpha},
\end{array}\right\}
$$

and the surface dilatation is

$$
e=\epsilon_{\alpha}+\epsilon_{\beta}=h^{2}\left(\frac{\partial u}{\partial \alpha}+\frac{\partial v}{\partial \beta}\right) .
$$

The rotation is

$$
\omega=\frac{h^{2}}{2}\left(\frac{\partial v}{\partial \alpha}-\frac{\partial u}{\partial \beta}\right)
$$


For plane stress and in the absence of body forces, $e /(1-\nu)+i \omega$ is a function of $z$, and hence of $w$, or

$$
\frac{e}{1-\nu}+i \omega=f(w)
$$

The stresses are related to the strains by Hooke's law, which for plane stress is

$$
\left.\begin{array}{rl}
\sigma_{\alpha} & =\frac{E}{1-\nu^{2}}\left(\epsilon_{\alpha}+\nu \epsilon_{\beta}\right), \\
\sigma_{\beta} & =\frac{E}{1-\nu^{2}}\left(\epsilon_{\beta}+\nu \epsilon_{\alpha}\right), \\
\tau_{\alpha \beta} & =\frac{E}{2(1+\nu)} \gamma_{\alpha \beta},
\end{array}\right\}
$$

where $E$ is Young's modulus of elasticity, and $\nu$ is Poisson's ratio.

\section{ELLIPTICAL COORDINATES}

Elliptical coordinates are obtained by writing for eq 15

$$
z=c \cosh w,
$$

from which

$$
x=c \cosh \alpha \cos \beta, \quad y=c \sinh \alpha \sin \beta .
$$

Elimination of $\beta$ from eq 24 gives

$$
\frac{x^{2}}{c^{2} \cosh ^{2} \alpha}+\frac{y^{2}}{c^{2} \sinh ^{2} \alpha}=1,
$$

a family of confocal ellipses with foci at $( \pm c, 0)$ and with semiaxes $c \cosh \alpha$ and $c \sinh \alpha$ (fig. 4). Elimination of $\alpha$ from eq 24 gives

$$
\frac{x^{2}}{c^{2} \cos ^{2} \beta}-\frac{y^{2}}{c^{2} \sin ^{2} \beta}=1,
$$

a family of hyperbolas confocal with and orthogonal to the ellipses of eq 25 (fig. 4 ).

For large $\alpha$, the elliptical coordinates $(\alpha, \beta)$ approach the polar coordinates $(r, \theta)$ in the following manner:

$$
\begin{aligned}
& \lim _{\alpha=\infty} \frac{\boldsymbol{c}}{2} e^{\alpha}=r, \\
& \lim _{\alpha=\infty} \beta=\theta .
\end{aligned}
$$

Eq 16 becomes

from which

$$
\frac{d z}{d w}=\frac{1}{h} e^{i \phi}=c \sinh w
$$

$$
h^{2}=\frac{2}{c^{2}(\cosh 2 \alpha-\cos 2 \beta)}
$$


314 Journal of Research of the National Bureau of Standards

and

$$
\tan \phi=\operatorname{coth} \alpha \tan \beta \text {. }
$$

Also,

$$
\begin{aligned}
& \frac{\partial h^{2}}{\partial \alpha}=-\frac{4}{c^{2}}-\frac{\sinh 2 \alpha}{(\cosh 2 \alpha-\cos 2 \beta)^{2}} \\
& \frac{\partial h^{2}}{\partial \beta}=-\frac{4}{c^{2}} \frac{\sin 2 \beta}{(\cosh 2 \alpha-\cos 2 \beta)^{2}}
\end{aligned}
$$

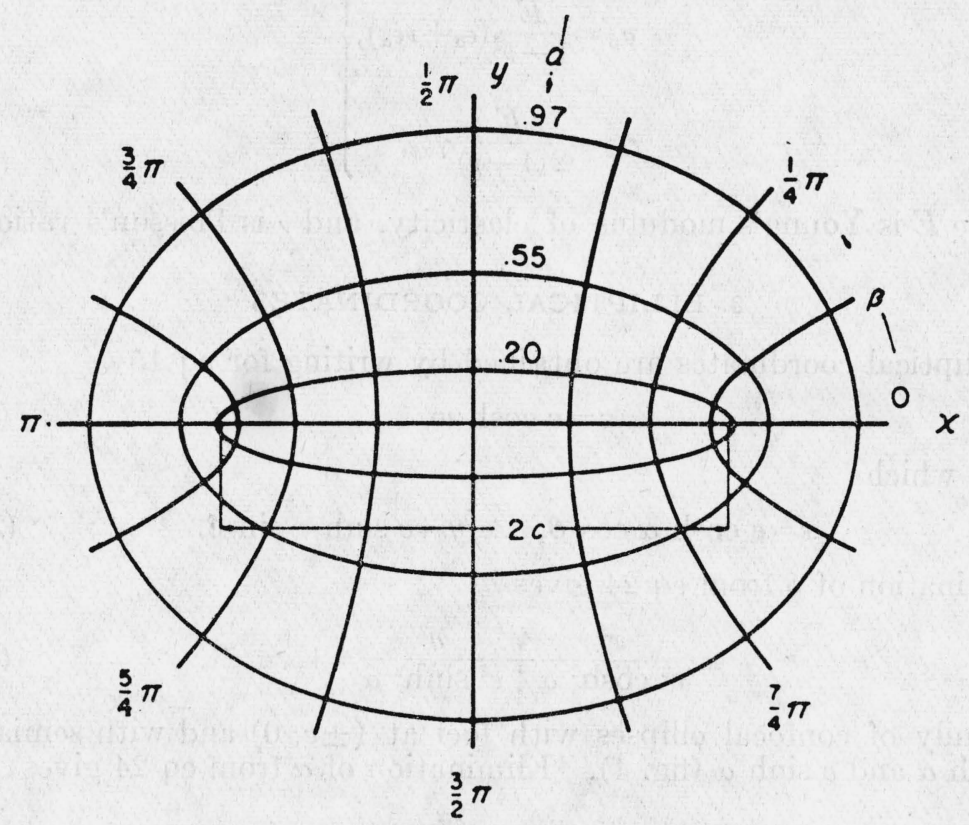

FIGURE 4.-Elliptical coordinates.

Denote by $\alpha=\alpha_{0}$ the ellipse

$$
\frac{x^{2}}{a^{2}}+\frac{y^{2}}{b^{2}}=1
$$

Then on the ellipse (eq 30 ), from eq 24 and 25 ,

$$
\left.\begin{array}{rlrl}
c \cosh \alpha_{0} & =a, & c \sinh \alpha_{0}=b, \\
\cos \beta & =\frac{x}{a}, & \sin \beta=\frac{y}{b}, \\
\cos 2 \beta & =\frac{x^{2}}{a^{2}}-\frac{y^{2}}{b^{2}}, & \sin 2 \beta & =\frac{2 x y}{a b} \\
\cosh 2 \alpha_{0} & =\frac{a^{2}+b^{2}}{c^{2}}, & e^{2 \alpha_{0}} & =\frac{a+b}{a-b}
\end{array}\right\}
$$


and from eq 27 and 31 ,

$$
\cos \phi=h_{0} \frac{b}{a} x, \quad \sin \phi=h_{0} \frac{a}{b} y,
$$

where $h_{0}$ is the value of $h$ on $\alpha=\alpha_{0}$.

\section{INGLIS' PROBLEM FOR PLANE STRESS}

The conditions of this problem may be satisfied by taking eq 21 as

$$
\frac{e}{1-\nu}+i \omega=c_{n} \frac{e^{-n w}}{\sinh w}
$$

where $n$ is any integer and $c_{n}$ is a real constant for any $n$. Substitution in eq 33 of the expressions for $e$ and $\omega$ given in eq 19 and 20 gives, upon equating real and imaginary parts,

$$
\begin{aligned}
& \frac{\partial u}{\partial \alpha}+\frac{\partial v}{\partial \beta}=\frac{c^{2}(1-\nu) c_{n}}{2}\left[e^{-(n-1) \alpha} \cos (n+1) \beta-e^{-(n+1) \alpha} \cos (n-1) \beta\right], \\
& \frac{\partial v}{\partial \alpha}-\frac{\partial u}{\partial \beta}=c^{2} c_{n}\left[-e^{-(n-1) \alpha} \sin (n+1) \beta+e^{-(n+1) \alpha} \sin (n-1) \beta\right] .
\end{aligned}
$$

The solution of eq 34 is

$$
\begin{aligned}
& u=a_{n}\left[(n+p) e^{-(n-1) \alpha} \cos (n+1) \beta+(n-p) e^{-(n+1) \alpha} \cos (n-1) \beta\right]+\phi, \\
& v=a_{n}\left[(n-p) e^{-(n-1) \alpha} \sin (n+1) \beta+(n+p) e^{-(n+1) \alpha} \sin (n-1) \beta\right]+\psi,
\end{aligned}
$$

where

$$
p=\frac{3-\nu}{1+\nu}
$$

$a_{\mathrm{n}}$ is constant for any $n$, and $u=\phi, v=\psi$, is the solution of the homogeneous equation corresponding to eq 34 . Suitable values of $\phi$ and $\psi$ are

$$
\phi=b_{m} e^{-m \alpha} \cos m \beta, \quad \psi=b_{m} e^{-m \alpha} \sin m \beta,
$$

where $m$ is any integer and $b_{m}$ is constant for any $m$. Hence

$$
\begin{gathered}
u=a_{n}\left[(n+p) e^{-(n-1) \alpha} \cos (n+1) \beta+(n-p) e^{-(n+1) \alpha} \cos (n-1) \beta\right] \\
+b_{m} e^{-m \alpha} \cos m \beta \\
v=a_{n}\left[(n-p) e^{-(n-1) \alpha} \sin (n+1) \beta+(n+p) e^{-(n+1) \alpha} \sin (n-1) \beta\right] \\
+b_{m} e^{-m \alpha} \sin m \beta .
\end{gathered}
$$

The strains may be calculated from eq 35 by means of eq 18 . These are given by

$c^{2}(\cosh 2 \alpha-\cos 2 \beta)^{2} \epsilon_{\alpha}=n a_{n}\left\{(n-2+p) e^{-(n-1) \alpha} \cos (n+3) \beta\right.$

$$
\begin{aligned}
& +(n+2-p) e^{-(n+1) \alpha} \cos (n-3) \beta \\
& -\left[(n+p) e^{-(n-3) \alpha}+2(p-1) e^{-(n+1) \alpha}\right] \cos (n+1) \beta \\
& \left.-\left[(n-p) e^{-(n+3) \alpha}-2(p-1) e^{-(n-1) \alpha}\right] \cos (n-1) \beta\right\} \\
& -b_{m}\left\{\left[(m+1) e^{-(m-2) \alpha}+(m-1) e^{-(m+2) \alpha}\right] \cos m \beta\right. \\
& \left.-(m+1) e^{-m \alpha} \cos (m-2) \beta-(m-1) e^{-m \alpha} \cos (m+2) \beta\right\},
\end{aligned}
$$


$c^{2}(\cosh 2 \alpha-\cos 2 \beta)^{2} \epsilon_{\beta}=n a_{n}\left\{-(n-p) e^{-(n-1) \alpha} \cos (n+3) \beta\right.$

$$
\begin{aligned}
& -(n+p) e^{-(n+1) \alpha} \cos (n-3) \beta \\
& +\left[(n+2-p) e^{-(n-3) \alpha}-2(p-1) e^{-(n+1) \alpha}\right] \cos (n+1) \beta \\
& \left.+\left[(n-2+p) e^{-(n+3) \alpha}+2(p-1) e^{-(n-1) \alpha}\right] \cos (n-1) \beta\right\} \\
& +b_{m}\left\{\left[(m+1) e^{-(m-2) \alpha}+(m-1) e^{-(m+2) \alpha}\right] \cos m \beta\right. \\
& \left.-(m+1) e^{-m_{\alpha}} \cos (m-2) \beta-(m-1) e^{-m \alpha} \cos (m+2) \beta\right\},
\end{aligned}
$$

$\frac{c^{2}}{2}(\cosh 2 \alpha-\cos 2 \beta)^{2} \gamma_{\alpha \beta}=n a_{n}\left\{(n-1) e^{-(n-1) \alpha} \sin (n+3) \beta\right.$

$$
\begin{aligned}
& +(n+1) e^{-(n+1) \alpha} \sin (n-3) \beta \\
& \left.-(n+1) e^{-(n-3) \alpha} \sin (n+1) \beta-(n-1) e^{-(n+3) \alpha} \sin (n-1) \beta\right\} \\
& -b_{m}\left\{\left[(m+1) e^{-(m-2) \alpha}+(m-1) e^{-(m+2) \alpha}\right] \sin m \beta\right. \\
& \left.-(m-1) e^{-m \alpha} \sin (m+2) \beta-(m+1) e^{-m \alpha} \sin (m-2) \beta\right\} .
\end{aligned}
$$

Substitution of these expressions in eq 22 gives for the stresses $(\cosh 2 \alpha-\cos 2 \beta)^{2} \sigma_{\alpha}=A_{n}\left\{(n+1) e^{-(n-1) \alpha} \cos (n+3) \beta\right.$

$$
\begin{aligned}
+ & (n-1) e^{-(n+1) \alpha} \cos (n-3) \beta \\
& -\left[(n+3) e^{-(n-3) \alpha}+4 e^{-(n+1) \alpha}\right] \cos (n+1) \beta \\
& \left.-\left[(n-3) e^{-(n+3) \alpha}-4 e^{-(n-1) \alpha}\right] \cos (n-1) \beta\right\} \\
+B_{m}\{ & -\left[(m+1) e^{-(m-2) \alpha}+(m-1) e^{-(m+2) \alpha}\right] \cos m \beta \\
+ & (m+1) e^{-m \alpha} \cos (m-2) \beta \\
+ & \left.(m-1) e^{-m \alpha} \cos (m+2) \beta\right\},
\end{aligned}
$$

$(\cosh 2 \alpha-\cos 2 \beta)^{2} \sigma_{\beta}=A_{n}\left\{-(n-3) e^{-(n-1) \alpha} \cos (n+3) \beta\right.$

$$
\begin{aligned}
&(n+3) e^{-(n+1) \alpha} \cos (n-3) \beta \\
&+ {\left[(n-1) e^{-(n-3) \alpha}-4 e^{-(n+1) \alpha}\right] \cos (n+1) \beta } \\
&\left.+\left[(n+1) e^{-(n+3) \alpha}+4 e^{-(n-1) \alpha}\right] \cos (n-1) \beta\right\} \\
&+B_{m}\left\{\left[(m+1) e^{-(m-2) \alpha}+(m-1) e^{-(m+2) \alpha}\right] \cos m \beta\right. \\
& \\
&-(m+1) e^{-m \alpha} \cos (m-2) \beta \\
&\left.-(m-1) e^{-m \alpha} \cos (m+2) \beta\right\},
\end{aligned}
$$

$(\cosh 2 \alpha-\cos 2 \beta)^{2} \tau_{\alpha \beta}=A_{n}\left\{(n-1) e^{-(n-1) \alpha} \sin (n+3) \beta\right.$

$$
\begin{aligned}
& +(n+1) e^{-(n+1) \alpha} \sin (n-3) \beta \\
& -(n+1) e^{-(n-3) \alpha} \sin (n+1) \beta \\
& \left.-(n-1) e^{-(n+3) \alpha} \sin (n-1) \beta\right\} \\
+B_{m}\{ & -\left[(m+1) e^{-(m-2) \alpha}+(m-1) e^{-(m+2) \alpha}\right] \sin m \beta \\
& +(m+1) e^{-m \alpha} \sin (m-2) \beta \\
& \left.+(m-1) e^{-m \alpha} \sin (m+2) \beta\right\}
\end{aligned}
$$

where

$$
A_{n}=\frac{n E}{(1+\nu) c^{2}} a_{n}, \quad B_{m}=\frac{E}{(1+\nu) c^{2}} b_{m} .
$$

This solution can be adapted to the case of an elliptical hole (eq 30) in a large plate subjected, at a large distance from the hole, to the uniform stresses $\sigma_{x}=S_{x}, \sigma_{y}=S_{y}, \tau_{x y}=0$. The boundary conditions in elliptical coordinates are

$$
\left.\begin{array}{c}
\sigma_{\alpha}=\tau_{\alpha \beta}=0,\left(\alpha=\alpha_{0}\right) ; \\
\sigma_{\alpha}=\frac{S_{x}+S_{y}}{2}+\frac{S_{x}-S_{y}}{2} \cos 2 \beta, \\
\sigma_{\beta}=\frac{S_{x}+S_{y}}{2}-\frac{S_{x}-S_{y}}{2} \cos 2 \beta \\
\tau_{\alpha \beta}=-\frac{S_{x}-S_{y}}{2} \sin 2 \beta,(\alpha=\infty) .
\end{array}\right\}
$$


These conditions can be satisfied by summing the solutions (eq 36) for $n=1,-1 ; m=2,0,-2$; with values for $A_{n}, B_{m}$,

$$
\left.\begin{array}{l}
A_{1}=\frac{e^{2 \alpha_{0}}}{8}\left(S_{x}-S_{y}\right)-\frac{1}{16}\left(S_{x}+S_{y}\right), \quad A_{-1}=-\frac{1}{16}\left(S_{x}+S_{y}\right), \\
B_{0}=-\frac{1}{4}\left(S_{x}-S_{y}\right)+\frac{\cosh 2 \alpha_{0}}{4}\left(S_{x}+S_{y}\right), \\
B_{2}=-\frac{e^{4 \alpha} 0}{8}\left(S_{x}-S_{y}\right), \quad B_{-2}=\frac{1}{8}\left(S_{x}-S_{y}\right) .
\end{array}\right\}
$$

The stresses are given by

$(\cosh 2 \alpha-\cos 2 \beta)^{2} \sigma_{\alpha}=\frac{S_{x}-S_{y}}{8}\left\{-\left[e^{2 \alpha}-2 e^{2 \alpha_{0}}+e^{2\left(2 \alpha_{0}-\alpha\right)}\right] \cos 4 \beta\right.$

$$
\begin{aligned}
& +\left[e^{4 \alpha}-4 e^{2\left(\alpha_{0}+\alpha\right)}+3\left(e^{4 \alpha_{0}}+1\right)-4 e^{2\left(\alpha_{0}-\alpha\right)}+e^{4\left(\alpha_{0}-\alpha\right)}\right] \cos 2 \beta \\
& \left.-e^{2 \alpha}+4 e^{2 \alpha_{0}}-\left(3 e^{4 \alpha_{0}}+2\right) e^{-2 \alpha}+2 e^{2\left(\alpha_{0}-2 \alpha\right)}\right\} \\
& +\frac{S_{x}+S_{y}}{2}\left(\cosh 2 \alpha-\cosh 2 \alpha_{0}\right) \sinh 2 \alpha
\end{aligned}
$$

$(\cosh 2 \alpha-\cos 2 \beta)^{2} \sigma_{\beta}=\frac{S_{x}-S_{y}}{8}\left\{\left[e^{2 \alpha}+2 e^{2 \alpha}{ }_{0}+e^{2\left(2 \alpha_{0}-\alpha\right)}\right] \cos 4 \beta\right.$

$$
\begin{aligned}
& -\left[e^{4 \alpha}+3\left(e^{4 \alpha}{ }_{0}+1\right)+8 e^{2\left(\alpha_{0}-\alpha\right)}+e^{4\left(\alpha_{0}-\alpha\right)}\right] \cos 2 \beta \\
& \left.+e^{2 \alpha}+4 e^{2 \alpha_{0}}+\left(3 e^{4 \alpha}{ }_{0}+2\right) e^{-2 \alpha}+2 e^{2\left(\alpha_{0}-2 \alpha\right)}\right\} \\
& +\frac{S_{x}+S_{y}}{2}\left(\cosh 2 a+\cosh 2 \alpha_{0}-2 \cos 2 \beta\right) \sinh 2 \alpha,
\end{aligned}
$$

$(\cosh 2 \alpha-\cos 2 \beta)^{2} \tau_{\alpha \beta}=\frac{S_{x}-S_{y}}{8}\left\{\left[e^{2 \alpha-} e^{2\left(2 \alpha_{0}-\alpha\right)}\right] \sin 4 \beta\right.$

$$
\begin{aligned}
& \left.-\left[e^{4 \alpha}+2 e^{2(\alpha+\alpha)}-3 e^{4 \alpha_{0}}-1+2 e^{2\left(\alpha_{0}-\alpha\right)}-e^{4\left(\alpha_{0}-\alpha\right)}\right] \sin 2 \beta\right\} \\
& +\frac{S_{x}+S_{y}}{2}\left(\cosh 2 \alpha-\cosh 2 \alpha_{0}\right) \sin 2 \beta .
\end{aligned}
$$

From eq 37 and 39 ,

$$
\begin{aligned}
& a_{1}=\frac{(1+\nu) c^{2}}{16 E}\left[2 e^{2 \alpha_{0}}\left(S_{x}-S_{y}\right)-\left(S_{x}+S_{y}\right)\right], \quad a_{-1}=\frac{(1+\nu) c^{2}}{16 E}\left(S_{x}+S_{y}\right), \\
& b_{0}=\frac{(1+\nu) c^{2}}{16 E}\left[4 \cosh 2 \alpha_{0}\left(S_{x}+S_{y}\right)-4\left(S_{x}-S_{y}\right)\right] \text {, } \\
& b_{2}=-\frac{(1+\nu) c^{2}}{16 E} 2 e^{4 \alpha_{0}}\left(S_{x}-S_{y}\right), \quad b_{-2}=\frac{(1+\nu) c^{2}}{16 E} 2\left(S_{x}-S_{y}\right) .
\end{aligned}
$$

Substitution of the constants (eq 41) into eq 35 gives, in consideration of eq 17 , for the displacements

$$
\begin{aligned}
\frac{8 E}{c^{2} h} u_{\alpha} & =\left(S_{x}-S_{y}\right)\left\{4 e^{2 \alpha_{0}} \cos 2 \beta-2(1-\nu) e^{2(\alpha} \theta_{\theta}^{-\alpha)}\right. \\
& \left.+(1+\nu)\left[\left(e^{2 \alpha}-e^{2\left(2 \alpha_{0}-\alpha\right)}\right) \cos 2 \beta-2\right]\right\} \\
& -2\left(S_{x}+S_{y}\right)\left[2 \cos 2 \beta-(1-\nu) \cosh 2 \alpha-(1+\nu) \cosh 2 \alpha_{0}\right],
\end{aligned}
$$


$\frac{8 E}{c^{2} h} u_{\beta}=-\left(S_{x}-S_{y}\right)\left\{2(1-\nu) e^{2 \alpha_{0}}+(1+\nu)\left[e^{2\left(2 \alpha_{0}-\alpha\right)}+e^{2 \alpha}\right]\right\} \sin 2 \beta$.

On the boundary $\alpha=\alpha_{0}$ the displacements $u_{\alpha}$ and $u_{\beta}$ of eq 42 assume the values

$u_{\alpha}=\frac{h_{0} c^{2}}{2 E}\left[\left(S_{x}-S_{y}\right)\left(e^{2 \alpha_{0}} \cos 2 \beta-1\right)+\left(S_{x}+S_{y}\right)\left(\cosh 2 \alpha_{0}-\cos 2 \beta\right)\right]$,

$u_{\beta}=-\frac{h_{0} c^{2}}{2 E}\left(S_{x}-S_{y}\right) e^{2 \alpha_{0}} \sin 2 \beta$.

The components of the displacement along the $x$ - and $y$-axes are obtained from eq 43 by the simple resolution

$$
\left.\begin{array}{l}
u_{x}=u_{\alpha} \cos \phi-u_{\beta} \sin \phi \\
u_{\nu}=u_{\alpha} \sin \phi+u_{\beta} \cos \phi .
\end{array}\right\}
$$

Substitution in eq 44 of the values of $u_{\alpha}$ and $u_{\beta}$ from eq 43 and of $\sin \phi$ and $\cos \phi$ from eq 32 gives, after reduction effected by means of eq 31

$$
\begin{gathered}
u_{x}=\left[\frac{a+b}{a}\left(S_{x}-S_{y}\right)+\frac{b}{a}\left(S_{x}+S_{y}\right)\right] \frac{x}{E}, \\
u_{y}=-\left[\frac{a+b}{b}\left(S_{x}-S_{y}\right)-\frac{a}{b}\left(S_{x}+S_{y}\right)\right] \frac{y}{E} .
\end{gathered}
$$

These are the rectangular components of the displacement on the bounding ellipse $\alpha=\alpha_{0}$, or $\left(x^{2} / a^{2}\right)+\left(y^{2} / b^{2}\right)=1$.

5. AXIAL RIGIDITY FOR LOAD PARALLEL TO MAJOR AXIS OF ELLIPSE

In this case the value to be substituted for $u_{p}$ in eq 2 is the value of $u_{x}$ from eq 45 with $S_{y}=0$, that is,

$$
u_{p}=\left(1+2 \frac{b}{a}\right) \frac{S_{x} x}{E} .
$$

It has been shown ${ }^{5}$ that the correction factor to be applied to the load $P$ of eq 2 is approximately independent of the shape of the elliptical hole. Hence, for most practical cases, $P$ is given by eq 11 (derived for a circular hole), i. e.,

where

$$
P=S_{x} A_{g}\left(1-\frac{1}{2 n^{2}}-\frac{1}{2 n^{4}}\right)=S_{x} A_{g} C(n),
$$

$$
n=\frac{A_{g}}{2 b t}=\frac{A_{g}}{A_{o}-A_{n}} .
$$

3 See p. 545 of reference [4]. 
Equation 2 becomes

$$
K=\frac{1}{1+\frac{1+2 \frac{b}{a}}{C(n)} \int x t d y}=\frac{1}{1+\frac{1+2 \frac{b}{a}}{C(n)} \frac{V_{0}}{V_{g}}}
$$

where $V_{0}$ is the volume of the perforation and $V_{\mathbf{B}}$ is the gross volume of one bay of the tension member.

6. AXIAL RIGIDITY FOR LOAD PARALLEL TO MINOR AXIS OF ELLIPSE

In this case the load is in the direction of the $y$-axis, and $d y$ in eq 2 should be replaced by $d x$. The value of $u_{p}$ is that of $u_{y}$ from eq 46 with $\mathrm{S}_{x}=0$, i. e.,

Again,

$$
u_{\nu}=\left(1+2 \frac{a}{b}\right) \frac{S_{\nu} y}{E}
$$

where

$$
P=S_{\nu} A_{g}\left(1-\frac{1}{2 n^{2}}-\frac{1}{2 n^{4}}\right)=S_{\nu} A_{g} C(n)
$$

and eq 2 becomes, finally,

$$
n=\frac{A_{g}}{2 a t}=\frac{A_{g}}{A_{g}-A_{n}}
$$

$$
K=\frac{1}{1+\frac{1+2 \frac{a}{b}}{C(n)} \frac{V_{0}}{V_{g}}}
$$

\section{TESTS ON TENSION MEMBERS HAVING CIRCULAR PERFORATIONS}

\section{GENERAL}

The approximations employed in the derivation of eq $12,13,48$, and 49 may be expected to introduce appreciable error if the perforations are too wide or if the spacing of the perforations is too small. Hence it was considered desirable to make some tests which would indicate the limits of the ranges of variables over which the formulas give satisfactory results.

Time for an extensive experimental investigation was not available; the work therefore was confined to the verification of eq 12 for circular holes.

For the case of a thin rectangular strip of width $w$ and thickness $t$, with a series of circular holes of diameter $d$ at midwidth and spaced uniformly $l$ on centers, eq 12 becomes

where $n=w / d$.

$$
\frac{1}{K}=1+\frac{3 \pi}{4\left(n-\frac{1}{2 n}-\frac{1}{2 n^{3}}\right) \frac{l}{d}},\left(\frac{l}{d}>1\right),
$$

It is easy to see that eq 50 will fail for sufficiently low $l / d$. The perforated strip cannot have an axial rigidity as low as that of an 
unperforated strip of width $w-d$, that is, $K$ must be greater than $(n-1) / n$. Yet eq 50 gives $K$ less than $(n-1) / n$ for

$$
1<\frac{l}{d}<\frac{2.3562}{1+\frac{1}{n}+\frac{1}{2 n^{2}}+\frac{1}{2 n^{3}}}
$$

The lower limit of $l / d$ (as a function of $n$ ) for which eq 50 is satisfactory is perhaps best determined by experiment. The same is true of $n$, since it is to be expected, from the method of derivation of eq 12 , that eq 50 will fail for sufficiently low $n$.

\section{DESCRIPTION}

Tests were made on strips, 26 in. long, $3 / 4$ in. wide, and 0.079 in. thick, of aluminum alloy $24 \mathrm{ST}$. There were four strips, one corresponding to each of the following values of $n: 5.84,3.87,2.92,1.94$.

The axial rigidity of each strip was measured before any holes had been made in it. Holes were then subdrilled in each strip and reamed to give the proper value of $n$. The holes were at midwidth of the strip and uniformly spaced along the length so that there were an integral number of bays in a 10-in. gage length. The axial rigidity of the perforated strip was measured, and then additional holes were made to halve the value of $l / d$, and so on successively until five tests had been made on each strip. The values of $l / d$ follow.

$$
\begin{aligned}
& n=5.84, l / d=\infty, 11.11,5.56,2.78,1.39 \\
& n=3.87, l / d=\infty, 12.92,6.46,3.23,1.61 \\
& n=2.92, l / d=\infty, 12.97,6.49,3.24,1.62 \\
& n=1.94, l / d=\infty, 12.95,6.48,3.24,1.62
\end{aligned}
$$

The stretch in a 10-in. gage length due to increasing the load from about $50 \mathrm{lb}$. to about $550 \mathrm{lb}$ was measured for each value of $l / d$. For a given $n$, the stretch for the unperforated strip $(l / d=\infty)$ divided by the stretch for a perforated strip gives the value of $K$ for the perforated strip.

The strips were held in Templin grips and loaded by dead weights (the same initial and final weights were used throughout) raised and lowered with the jack of an Amsler vertical hydraulic testing machine. The stretch was measured using a pair of 10-in. gage-length Tuckerman optical strain gages provided with either 0.2 -in. or 0.5 -in. lozenges.

The permanent set produced by the 550-lb load was negligible in each case.

The test setup is shown in figure 5.

\section{RESULTS}

The results of the tests and a comparison of the observed with the theoretical values of $K$ are shown in figure 6 .

For $l / d$ greater than about 3 , the error in the theoretical $K$ increases with decreasing $n$ and is about 0.02 to 0.03 for $n$ about 2 .

For small $l / d$, the theoretical $K$ is too low, especially for large $n$. 


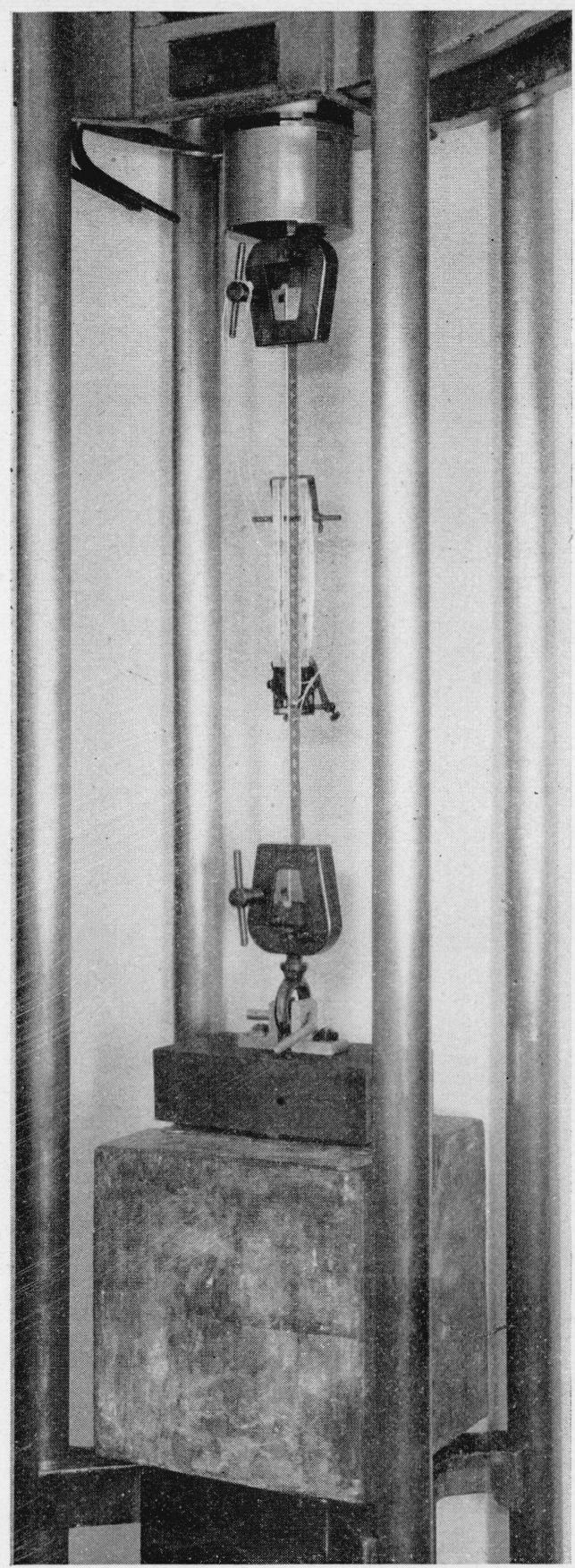

Figure 5.-General view of test setup. 
These results indicate that eq 50 can be relied upon to give $K$ within 0.03 for $n>2, l / d>2$, and within perhaps 0.015 for $n>3, l / d>3$

\section{SUMMARY}

Approximate formulas have been developed for the computation of the axial rigidity of a long tension or compression member containing a plate of constant thickness uniformly perforated with a series of circular, elliptical, or ovaloid holes.

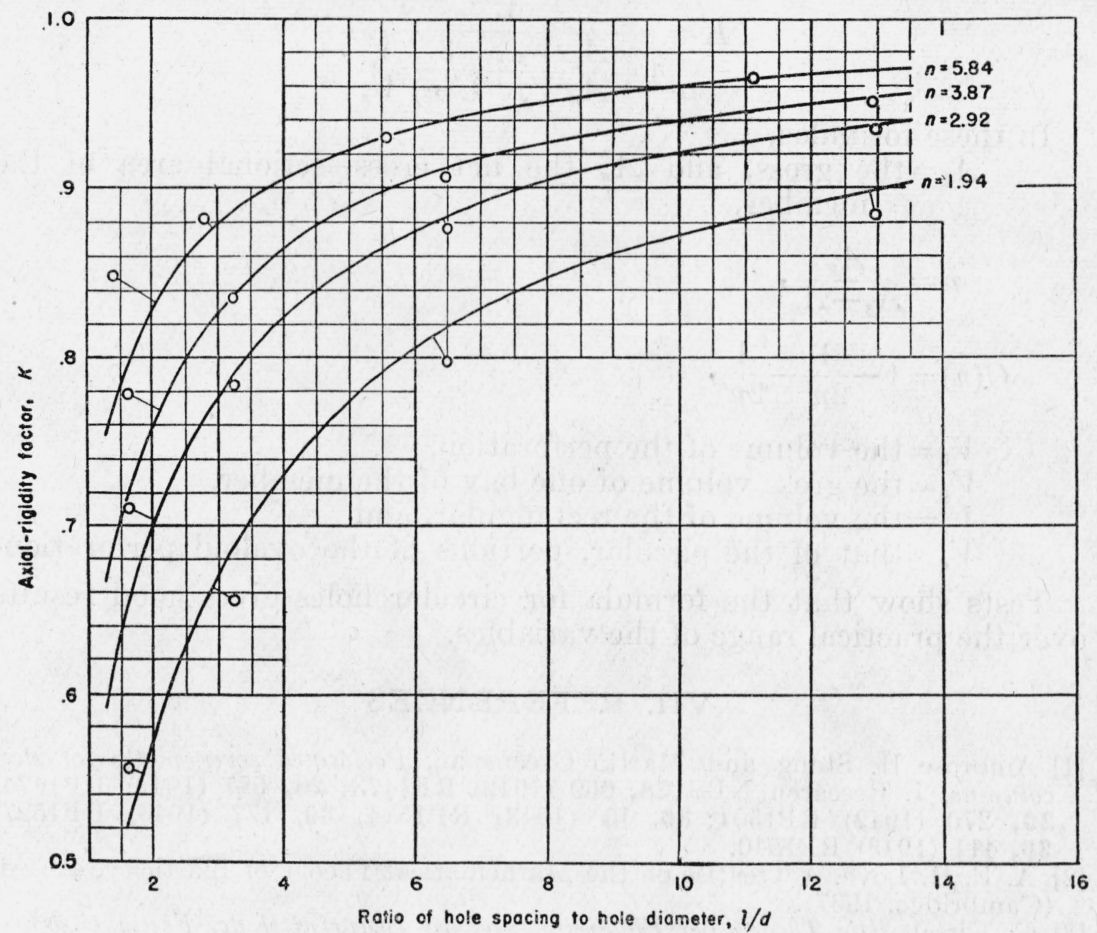

Figure 6.-Variation of $K$ with $n$ and $l / d$

The curves show the theoretical values (eq 50), the circles the observed values.

The axial-rigidity factor $K$ is defined so that $K E A_{g}$ is the rigidity which should be used in place of $E A_{g}$ in the ordinary formula for computation of the extension of the member.

The formulas for $K$ are:

for circular holes,

$$
K=\frac{1}{1+\frac{3}{C(n)} \frac{V_{0}}{V_{g}}}
$$

for elliptical holes of semimajor axis $a$ and semiminor axis $b$,

$$
K=\frac{1}{1+\frac{1+2 \frac{b}{a}}{C(n)} \frac{V_{0}}{V_{g}}},
$$


if the load is parallel to the major axis, and

$$
K=\frac{1}{1+\frac{1+2 \frac{a}{b}}{C(n)} \frac{V_{0}}{V_{g}}},
$$

if the load is parallel to the minor axis of the ellipse; and for ovaloid holes

In these formulas,

$$
K=\frac{1}{1+\frac{A_{g}}{A_{n}} \frac{V_{r}}{V_{g}}+\frac{3}{C(n)} \frac{V_{c}}{V_{g}}} .
$$

$A_{g}=$ the gross, and $A_{n}$ the net cross-sectional area of the member,

$$
n=\frac{A_{g}}{A_{g}-A_{n}}
$$

$$
C(n)=1-\frac{1}{2 n^{2}}-\frac{1}{2 n^{4}}
$$

$V_{0}=$ the volume of the perforation,

$V_{g}=$ the gross volume of one bay of the member,

$V_{r}=$ the volume of the rectangular, and

$V_{c}=$ that of the circular, portions of the ovaloid perforation.

Tests show that the formula for circular holes gives good results over the practical range of the variables.

\section{REFERENCES}

[1] Ambrose H. Stang and Martin Greenspan, Perforated cover plates for steel columns, J. Research NBS 28, 669 (1942) RP1473; 28, 687 (1942) RP1474; 29, 270 (1942) RP1501; 30, 15 (1943) RP1514; 30, 177 (1943) RP1527; 30, 411 (1943) RP1540.

[2] A. E. H. Love, A Treatise on the Mathematical Theory of Elasticity, 4th ed. (Cambridge, 1937).

[3] G. Kirsch, Die Theorie der Elastizität und die Bedürfnisse der Festigkeitslehre. Z. Ver. deut. Ing. XXXXII, 797 (1898).

[4] E. G. Coker and L. N. G. Filon, A Treatise on Photoelasticity (Cambridge 1931).

5] C. E. Inglis, Stresses in a plate due to the presence of sharp corners. Trans. Inst. Naval Arch. LV, 219 (1913, I).

INGToN, September 22, 1943. 